
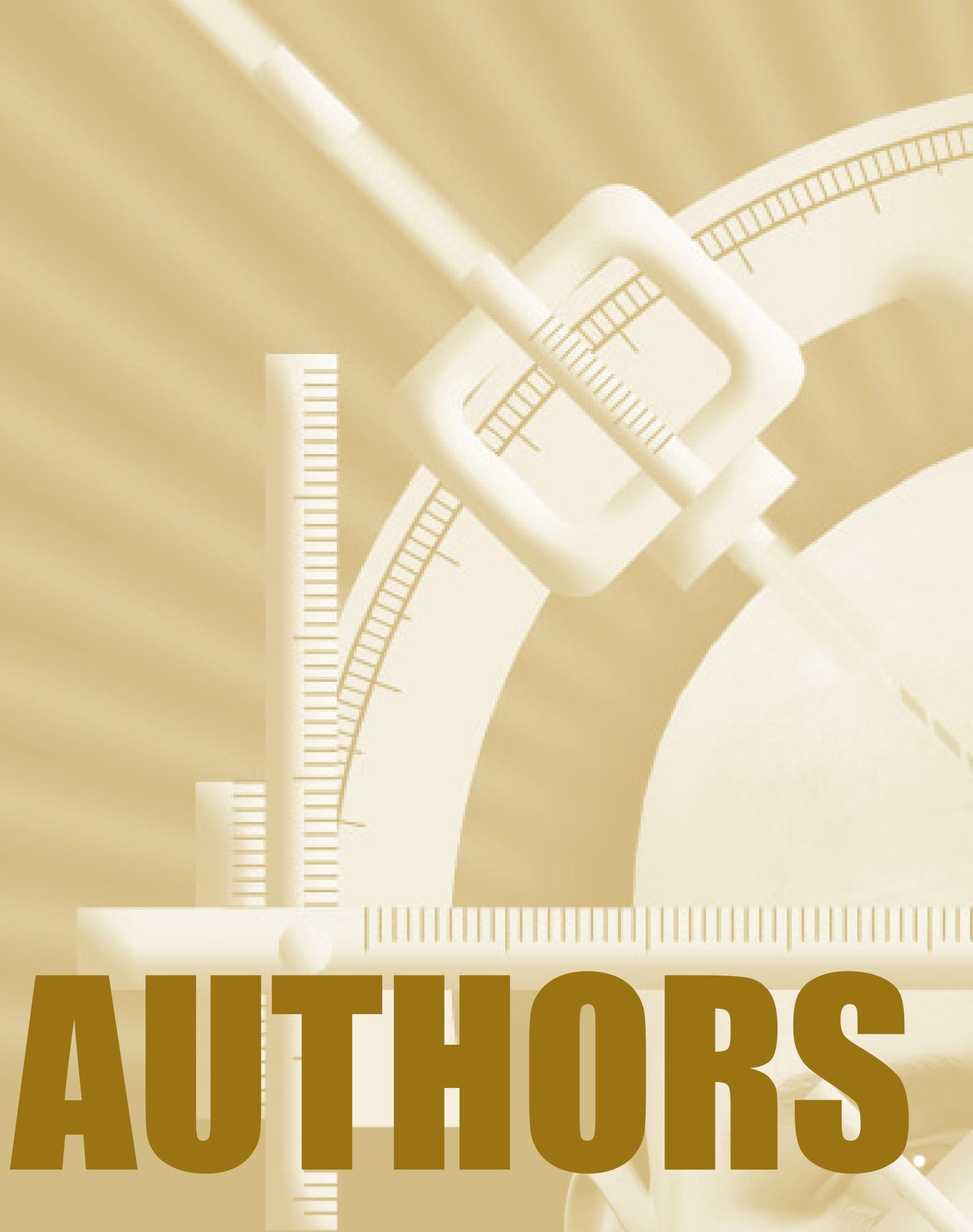


\section{INDEK OF AUTHORS}

A

Abdallat, M. • MOVDIS/P/222

Abosch, A. • RESIND/O/178, RESIND/O/159

Acar, F. • MOVDIS/F/108, PAIN/O/109, MOVDIS/P/107

Acar, G. • MOVDIS/F/108, MOVDIS/P/107

Acedo, M.V. • BSIDT/O/230

Ackermans, L. • MOVDIS/F/125

Aerts, J.M. • PSYSUR/O/279

Afshar, P. • RESIND/O/143

Akcakaya, M. O. • PAIN/O/196

Akdemir, G. • MOVDIS/P/50

Al Helli, O - MOVDIS/F/56

Alam, M. • BSIDT/O/102, MOVDIS/O/106, MOVDIS /F/101, MOVDIS/F/97, PSYSUR/P/219

Alesch, F. • BSIDT/O/309, MOVDIS/P/247

Alessi, G. • BSIDT/O/71, BSIDT/P/69, BSIDT/P/70

Almeida, A. • RADSUR/O/154

Almqvist, P. • PSYSUR/O/202, PAIN/P/204

Alonso, P. • BSIDT/O/230

Amaro, Jr. E. • BSIDT/P/58, BSIDT/P/59

Anasetti, F. • BSIDT/F/276

Andersson, M. • PSYSUR/F/205

Andreadis, N. • BSIDT/O/263, BSIDT/P/264

Angelakis, E. • BSIDT/O/263, BSIDT/P/264

Angelov, S. • BSIDT/O/102, PSYSUR/P/99

Antonini, A. • MOVDIS/P/83, MOVDIS/P/61, MOVDIS/P/62

Appenrodt, P. • RESIND/O/111

Aran-Echabe,E. • MOVDIS/P/209

Arango, G. • MOVDIS/F/182, MOVDIS/F/184, PSYSUR/O/181, MOVDIS/P/260

Aras, Y. • PAIN/O/196

Arias, J. A. • BSIDT/P/250

Arienta, C. B BSIDT/F/253

Arle, J. P PAIN/O/42, PAIN/O/43

Arle, J. E. • MOVDIS/O/121

Arza, R. • PSYSUR/P/233

Aschermann, Z. • MOVDIS/F/122

Ashkan, K. • MOVDIS/F/243, OTHER/P/239

Asplund, P. • PAIN/O/79

Astradsson, A. • PSYSUR/P/262, RADSUR/P/176

Åström, M. • MOVDIS/O/73, MOVDIS/O/74, PSYSUR/F/205

Aumüller, M. • MOVDIS/P/217

Avecillas, J. • BSIDT/O/230, PSYSUR/P/233

Ayaz, M. $\cdot$ MOVDIS/P/50

Aydoseli, A. • PAIN/O/196

Aygun, D. • MOVDIS/P/80

Ayres Basto, M. $\cdot$ MOVDIS/F/211

Azulay, J.-P. • MOVDIS/O/166, MOVDIS/O/271, MOVDIS/F/272 
B

Babos, M. • MOVDIS/P/224

Baillif, S. • PAIN/P/52

Bakay, R. • BSIDT/O/33

Balanescu, B. • BSIDT/P/68

Balas, I. • MOVDIS/F/122

Balugo, P. • BSIDT/O/230

Barborica, A. • BSIDT/P/68

Barbosa, M. • PAIN/F/117

Barcella, A. • MOVDIS/P/62

Barcia, J. A. • BSIDT/O/230, PSYSUR/P/233

Barlas, O. • PAIN/O/196

Barszcz, S. • MOVDIS/F/55

Bartolomei, F. • EPIL/O/131, EPIL/O/72

Barzó, P. • MOVDIS/P/224

Basso, G. • BSIDT/F/294

Bastianello, S. • BSIDT/F/253

Battaglia, G. • RADSUR/O/75

Bayrakli, F. • PAIN/O/109

Beck, J. • BSIDT/F/139

Beckers, M. · MOVDIS/O/290

Belloch, V. • BSIDT/F/153

Belo, F. P PAIN/F/117

Benabid, A. L. • PAIN/O/228

Bender, B. • MOVDIS/O/85

Benedetti, J. • PSYSUR/O/181

Bengel, F. • PSYSUR/O/226

Bentes, C. • EPIL/O/142, EPIL/O/246

Berding, G. • PSYSUR/O/226, MOVDIS/P/222

Bergenheim, T. P PAIN/O/79

Bergman, H. • MOVDIS/O/51

Bernath, D. • BSIDT/P/307

Beute, G. • MOVDIS/O/274, PAIN/F/93, PSYSUR/F/94

Bewernick, B. • PSYSUR/O/293

Bhatia, R. • MOVDIS/F/300, MOVDIS/P/298

Bianchi Marzoli, S. • RADSUR/O/147

Bickel, S. • EPIL/O/266

Biella, G. • BSIDT/F/253

Bilbao, G. • MOVDIS/O/113

Bir, L. S. • MOVDIS/F/108

Blahak, C. • MOVDIS/F/76, MOVDIS/F/77, MOVDIS/P/78

Bloch, J. • MOVDIS/P/86, MOVDIS/P/57

Blokland, A. • PSYSUR/O/123

Blomstedt, P. • MOVDIS/O/73, MOVDIS/O/74, PSYSUR/F/197, MOVDIS/P/288

Blond, S. · BSIDT/O/286, RADSUR/P/267

Bob, P. • MOVIS/F/241

Boetto, J. • MOVDIS/O/232, MOVDIS/F/198

Boex, C. E EPIL/O/163

Bogiel, T. • BSIDT/F/326

Bolognesi, A. • RADSUR/O/147

Bondallaz, P. • EPIL/O/163 


\section{INDEK OF AUTHORS}

Bóné, B. • MOVDIS/F/122

Bonicki, W. • MOVDIS/F/55, MOVDIS/P/54

Bosch, A. • PSYSUR/O/45

Bosnyák, E. • MOVDIS/F/122

Bot, M. • BSIDT/O/33

Botelho, L. • MOVDIS/F/177

Botella, C. B BSIDT/F/153

Bötzel, K. • MOVDIS/O/252

Boutos, N. • MOVDIS/O/192, MOVDIS/F/195

Brás, J. • MOVDIS/F/212, RADSUR/F/229, MOVDIS/P/245, PAIN/P/207

Bratsky, L. • EPIL/P/240

Bravin, A. • RADSUR/O/75

Breit, S. $\cdot$ MOVDIS/O/85

Brin, J. R. • BSIDT/O/230

Brionne, T. $\cdot$ RESIND/O/111

Brodacki, B. • MOVDIS/P/54

Broggi, G. • BSIDT/O/270, MOVDIS/O/135, RADSUR/O/116

Brouns, R. • BSIDT/F/318

Brücke, C. • PSYSUR/F/235

Bruecke, T. • MOVDIS/P/247

Bühler, R. • BSIDT/O/297

Burchiel, K. J. • PAIN/O/109

Buschman, R. • RESIND/O/111

Bustamante, J. A. • PSYSUR/F/249

Butler, A. • RESIND/O/144

Butson, C. R. • MOVDIS/O/121

1

Cabañes, L. • MOVDIS/F/105

Cabral, J. • EPIL/F/238

Cabrita, F. • PAIN/F/117

Calado, C. • MOVDIS/P/245

Campos, J. $\cdot$ RADSUR/O/154, BSIDT/P/250

Capecci, M. • MOVDIS/O/98, MOVDIS/F/100, EPIL/P/103

Capelle, H.-H. • MOVDIS/O/221, MOVDIS/O/106, MOVDIS/F/76, MOVDIS/F/77, PSYSUR/O/226, PSYSUR/F/235, MOVDIS/P/78

Capone, J. $\cdot$ MOVDIS/F/295

Carcieri, S. • RESIND/O/206, MOVDIS/P/247

Cardoso, P. • BSIDT/O/138

Carena, P. • BSIDT/F/168

Carlson, K. P PAIN/O/43

Carlson, T. $\cdot$ RESIND/O/144

Carrillo, J. D. • PSYSUR/F/249, PSYSUR/F/34, PSYSUR/P/251

Carro, M. S. • BSIDT/F/326

Carron, R. • EPIL/O/72, MOVDIS/O/165, MOVDIS/O/166, MOVDIS/O/271, MOVDIS/F/272, PAIN/O/187, PAIN/O/127, PAIN/O/228, RADSUR/O/129

Carvalho, M. · PAIN/F/117

Castro, G. MOVDIS/O/87

Castro-García, A. - MOVDIS/P/209

Cattoni, M. B. • MOVDIS/O/254, MOVDIS/F/255, RADSUR/F/229

Cavallo, M. A. • BSIDT/F/294, MOVDIS/F/295 
Ceravolo, M. G. • MOVDIS/O/98, MOVDIS/F/100, EPIL/P/103

Ceyssens, F. • BSIDT/O/278, PSYSUR/O/279

Chabardès, S. • EPIL/O/237, PAIN/O/228

Chamadoira, C. • MOVDIS/F/211, MOVDIS/P/303

Charrier, R. • BSIDT/P/216

Chastan, N. $\cdot$ MOVDIS/O/63

Chauvel, P. • EPIL/O/131, EPIL/O/72

Chen, S.-Y. • MOVDIS/P/112, MOVDIS/P/164

Chiari, L. • MOVDIS/F/295

Chiou, S.-M. $\cdot$ MOVDIS/P/175

Chivoret, N. • EPIL/O/237

Cho, C. B. • MOVDIS/P/67

Cho, K.-S. P PAIN/P/265

Chough, C. K. • PAIN/P/26

Chowdhury, Y. • BSIDT/P/306

Chudy, D. • BSIDT/F/172

Chung, S. S. • PAIN/P/179

Cif, A. L. • MOVDIS/O/232, MOVDIS/O/104, MOVDIS/F/148, PSYSUR/F/118, PSYSUR/P/92

Cirak, B. • PAIN/O/109

Ciurea, J. • BSIDT/P/68

Cividini, A. • PAIN/F/268

Claus, R. • BSIDT/F/326

Coelho, M. • MOVDIS/O/254, MOVDIS/F/255

Coenen, V. A. • PSYSUR/O/293

Colle, D. • BSIDT/O/71, BSIDT/P/69, BSIDT/P/70

Colle, H. • BSIDT/O/71, BSIDT/P/69, BSIDT/P/70

Concha, L. • MOVDIS/O/87

Conde, R. B BSIDT/F/153

Condeça, B. • MOVDIS/F/141

Contreras Lopez, W. O. • BSIDT/F/305, BSIDT/P/259, MOVDIS/P/260, RADSUR/P/261

Cordeiro, I. • MOVDIS/F/141

Cordeiro, J. G. • BSIDT/O/161, RADSUR/P/130, OTHER/P/287

Cordeiro, K. K. • BSIDT/O/161

Cordella, R. • BSIDT/O/270

Correia, F. • BSIDT/O/236

Correia, I. • BSIDT/O/138

Coskun, E. PAIN/O/109

Costa, G. • PAIN/F/117

Costa, H. • MOVDIS/P/303

Coste, J. · MOVDIS/P/95

Coubes, P. • BSIDT/O/114, MOVDIS/O/232, MOVDIS/O/104, MOVDIS/F/198, PSYSUR/F/118, PSYSUR/P/92

Coutinho Escolástico, A. R. • RADSUR/O/154

Cristiani, P. • EPIL/F/189

Csaszar, N. • BSIDT/P/307

Csigó, K. • PSYSUR/O/244

Csókay, A. • MOVDIS/P/190

I

Dai, F. • BSIDT/F/326

Dani, S. • RESIND/O/144 
Daniel, R. • RADSUR/P/186

Daquin, G. $\cdot$ EPIL/O/131

Darcourt, J. • PSYSUR/P/231

Dario, A. • PAIN/F/268

da Silva, R. E. • BSIDT/P/59

D'Auria, S. • BSIDT/F/119

David, O. • EPIL/O/237

De Benedictis, A. • BSIDT/F/294

De Bie, R. • MOVDIS/O/274

De Fazio, P. • PSYSUR/O/282

De Rose, M. • PSYSUR/O/282

De Smedt, A. • BSIDT/F/318

Dekopov, A. • MOVDIS/O/312, MOVDIS/F/273

Del Álamo • MOVDIS/F/105

Del Vecchio, A. • RADSUR/O/147

Deletis, V. • BSIDT/F/172

Delfini, M. • MOVDIS/O/271

Deli, G. • MOVDIS/F/122

Demeter, G. • PSYSUR/O/244

Denison, T. • RESIND/O/143, RESIND/O/144

Denys, D. • PSYSUR/O/45

Derrey, S. • MOVDIS/O/63

De Smeolt, A. BSIDT/F/318

Deudon, A. • PSYSUR/P/231

Deuschl, G. • MOVDIS/P/223

Dézsi, L. • MOVDIS/P/224

D'Haen, B. • BSIDT/O/71, BSIDT/P/69, BSIDT/P/70

D'Hardemare, V. • RADSUR/P/267

Di Meco, F. • RADSUR/O/116

Diaz-Cabanas, L. • MOVDIS/P/209

Diczfalusy, E. • PSYSUR/F/205

Diserens, K. • MOVDIS/P/57

Döbrössy, M. • BSIDT/O/161, BSIDT/F/326, MOVDIS-T/O/313

Dóczi, T. • MOVDIS/F/122

Dolgun, M. • PAIN/O/196

Dones, I. · BSIDT/O/270, MOVDIS/O/135, PAIN/P/49

Donnet, A. • PAIN/O/187, PAIN/O/127, PAIN/O/126, PAIN/P/52

Donos, C. • BSIDT/P/68

Doostkam, S. • BSIDT/F/326

dos Santos, G. A. B. • BSIDT/P/59

Dragasek, J. • EPIL/P/240

Droogmans, S. • BSIDT/F/318

Dubois, F. • BSIDT/O/286

Dubrulle, F. • RADSUR/P/267

Duffau, H. • BSIDT/F/294

Dura, J. L. • BSIDT/F/153

Duru, C. B BSIDT/F/47

Dzierzecki, S. • MOVDIS/F/32, MOVDIS/P/29, MOVDIS/P/30, MOVDIS/P/31 


\section{INDEK OF AUTHORS}

\section{E}

Eitan, R. $\cdot$ MOVDIS/O/51

Ekmekci, H. • MOVDIS/P/50

El Hassani, Y.・ EPIL/O/162

El Majdoub, F. • BSIDT/O/302, RADSUR/O/301

Eleopra, R. • BSIDT/F/119

Elias, W. J. • MOVDIS/O/121

Eljamel, S. • BSIDT/F/168, MOVDIS/O/40, PAIN/P/41

Entz, L. • EPIL/O/266, BSIDT/P/307, PAIN/P/304

Erisksdotter, M. • PSYSUR/O/202

Eross, L. • EPIL/O/266, BSIDT/P/307, PAIN/P/304

Escalante Higuera, C. A. • BSIDT/F/305

Escobar, O. • MOVDIS/F/182, MOVDIS/F/184, PSYSUR/O/181, MOVDIS/P/260

Espinoza Martinez, J. A. • BSIDT/F/305

Espinoza, J. • MOVDIS/F/182, MOVDIS/F/184, PSYSUR/O/181, MOVDIS/P/260

Eusebio, A. • MOVDIS/F/272

Evers, S. $\cdot$ MOVDIS/F/125

Eyjolfsdottir, H. • PSYSUR/O/202

Ezquiaga, E.・PSYSUR/O/64

\section{F}

Fabó, D. • EPIL/O/266

Falk, D. • MOVDIS/O/208, MOVDIS/P/223, MOVDIS/P/210

Fardone, E. • RADSUR/O/75

Fariselli, L. • RADSUR/O/116

Fedorova, N. • MOVDIS/O/312

Fehrmann, E.・RESIND/O/144

Feketeova, E.・EPIL/P/240

Feldt-Rasmussen, U. • RADSUR/P/176

Felgueiras, R. • MOVDIS/F/177

Ferraresi, G. • MOVDIS/F/295

Ferrari Da Passano, C. • RADSUR/O/147

Ferreira, J. • MOVDIS/O/254, MOVDIS/F/255

Ferroli, P. • RADSUR/O/116

Figee, M. • PSYSUR/O/45

Filipchuk, A. • MOVDIS/O/165

Firat, E. B BSIDT/F/326

Firat Ince, N. • RESIND/O/159, RESIND/O/178

Fluchère, F. • MOVDIS/F/272

Foki, T. $\cdot$ MOVDIS/F/46

Foltynie, T. • BSIDT/F/167, MOVDIS/F/56, PSYSUR/F/205

Fonoff, E. T. • BSIDT/P/58, BSIDT/P/59

Fontaine, D. • EPIL/O/237, BSIDT/P/242, PAIN/P/52, PSYSUR/P/231

Foote, K. D. • MOVDIS/O/121

Forsgren, L. • MOVDIS/O/74

Franzini, A. • BSIDT/O/270, MOVDIS/O/135, PSYSUR/O/282, RADSUR/O/116, RADSUR/O/147, PAIN/P/49

Fréger, P. • MOVDIS/O/63

Freund, H.-J - PSYSUR/O/110

Fritz, M. • MOVDIS/O/85

Fytagoridis, A. • MOVDIS/O/73, MOVDIS/O/74 


\section{INDEK OF AUTHORS}

G

Gabrecht, T. • RESIND/O/111

Galanda, M. • MOVDIS/F/241

Galanda, T. • MOVDIS/F/241

Gamaleya, A. • MOVDIS/O/312, MOVDIS/F/241

Gambini, O. • PSYSUR/O/282

García de Sola, R. • EPIL/O/65, PSYSUR/O/64, EPIL/P/155

Garcia, D. • MOVDIS/O/84

Garcia, G. • MOVDIS/O/87

Garcia, J. • BSIDT/O/161

García-Albea, J. • PSYSUR/P/233

García-Caldentey, J. • MOVDIS/F/105

García-Camba, E. • PSYSUR/O/64

García de Sola, R. G. • PSYSUR/O/64

García-Navarrete, E. • EPIL/O/65

Gatterbauer, B. • MOVDIS/F/46

Gatzonis, S. • MOVDIS/O/192, MOVDIS/F/195

Gaudart, J. • PAIN/O/187

Gedik, B. • MOVDIS/F/108

Gelabert-Gonzalez, M. • MOVDIS/P/209

Getschmann, M. • BSIDT/O/102

Geworski, L. • PSYSUR/O/226

Gharabaghi, A. • MOVDIS/O/85

Giannicola, G. P PSYSUR/P/291

Giraud, P. • PAIN/O/228

Gmünder, D. • MOVDIS/P/95

Goebel, S. • MOVDIS/P/210

Goetz, S. • RESIND/ O/145, RESIND/O/146

Gomes, F. • PAIN/F/117

Gómez, J. C. • MOVDIS/O/113

Gonçalves-Ferreira, A. • BSIDT/O/138, BSIDT/O/236, EPIL/O/142, EPIL/O/246, MOVDIS/O/254, MOVDIS/F/141, MOVDIS/F/255, PSYSUR/O/136, RADSUR/O/154

Gonçalves fereira, D. P PSYSUR/O/136

González, G. • MOVDIS/F/248

Gonzalez, V. • MOVDIS/O/232, MOVDIS/O/104, MOVDIS/F/198, PSYSUR/F/118, PSYSUR/P/92

Gonzalez-Hidalgo, M. • BSIDT/O/230

Górecki, W. • MOVDIS/F/32, MOVDIS/P/29, MOVDIS/P/30, MOVDIS/P/31

Gornik Kramberger, K. • BSIDT/P/319

Gottweis, H. • MOVDIS-T/O/314

Gourcerol, G. • MOVDIS/O/63

Grahovac, G. • BSIDT/F/172

Granieri, E.・BSIDT/F/294, MOVDIS/F/295

Grinberg, I. • MOVDIS/P/223

Grinberg, L. T. • BSIDT/P/58, BSIDT/P/59

Guedes, L. • MOVDIS/O/254, MOVDIS/F/255

Gupta, R. • RESIND/O/159

Guridi, J. • MOVDIS/O/84

Gutierrez-Martin, A. • BSIDT/F/153

Guz, H. • MOVDIS/P/80 


\section{INDEK OF AUTHORS}

H

Haag, D. • BSIDT/P/216

Haapasalo, J. • EPIL/O/277

Haegelen, C. • MOVDIS/P/247

Hamberg, K. • OTHER/P/225

Hammond, C. • MOVDIS/O/165

Harat, D. • BSIDT/P/296

Hardenacke, K. • PSYSUR/O/110

Hariz, G.-M. • OTHER/P/225

Hariz, M. • BSIDT/O/114, BSIDT/F/167, MOVDIS/O/74, MOVDIS/F/300, MOVDIS/F/56, PSYSUR/O/66, PSYSUR/F/197, PSYSUR/F/205, MOVDIS/P/298, OTHER/P/225

Harries, A. • MOVDIS/O/213, MOVDIS/O/214

Harsányi, A. • PSYSUR/O/244

Hasegawa, H. • MOVDIS/F/243, OTHER/P/239

Heinsen, H. • BSIDT/P/58, BSIDT/P/59

Heissler, H. • MOVDIS/O/221, MOVDIS/O/106, PSYSUR/P/219

Hemm-Ode, S. • BSIDT/O/90, MOVDIS/P/95

Hennerici, M. G. • MOVDIS/F/76, MOVDIS/F/77

Henriksen, T. • PSYSUR/P/262

Herculano Carvalho, M. • EPIL/O/142, EPIL/O/246, MOVDIS/O/254, MOVDIS/F/255, RADSUR/O/154

Herold-Mende, C. • BSIDT/F/326

Hescham, S. P PSYSUR/O/123

Hoffmann, C. MOVDIS/O/274

Holton, J. • MOVDIS/F/56

Huebl, J. • PSYSUR/F/235

Huh, P. W. PAIN/P/265

Huh, R. PAIN/P/179

Hung, K. N. • RADSUR/P/171

Hutter Celik, S. • BSIDT/P/319

Iftimia, N. • PAIN/O/43

Ikbal, S. • RADSUR/F/229

Illés, Z. • MOVDIS/F/122

Imberti, R. • BSIDT/F/253

Ince, N. • RESIND/O/159, RESIND/O/178

Inoue, H. • RADSUR/O/91

lotti, G. BSIDT/F/253

Isagulyan, E. • MOVDIS/O/312, PAIN/O/257, PAIN/P/258

Ishizuka, N. • MOVDIS/P/193

Israel, Z. • MOVDIS/O/51

Iwasaki, K.・BSIDT/P/311

J

Jackson, A. • RESIND/O/206

Jacobsen Teixeira, M. • MOVDIS/O/220

Jahanshahi, A. • BSIDT/O/124, PSYSUR/O/123 
Jain, R. • MOVDIS/P/247

Jacobsen Teixeira, M. $\cdot$ MOVDIS/O/220

James, S. • MOVDIS/O/232, MOVDIS/O/104, MOVDIS/F/198, PSYSUR/F/118, PSYSUR/P/92

Jamieson, G. O OTHER/P/239

Jang, J. $\cdot$ RADSUR/P/27

Janssen, M. • BSIDT/O/124

Janszky, J. • MOVDIS/F/122

Jasova, D. • MOVDIS/F/241

Jeanmonod, D. • BSIDT/O/297

Jech, R. • MOVDIS/F/149

Jenkinson, M. • MOVDIS/P/224

Jenny, B. • EPIL/O/162

Jespersen, B. • PSYSUR/P/262

Jeyalan, V. • MOVDIS/O/40

Jiang, J.-L. • MOVDIS/P/164

Jiménez, F. • MOVDIS/F/248, PSYSUR/F/249, PSYSUR/F/34, BSIDT/P/250, PSYSUR/P/251

Jin, S.-T. • MOVDIS/P/185, PAIN/P/183

Jobbágy, Á - MOVDIS/O/191, MOVDIS/P/190

Johnsen, E. L. • MOVDIS/F/173, MOVDIS/F/174

Jombik, P. • MOVDIS/F/241

Joo, W.-I. • PAIN/P/26

Jourdain, V. A. • MOVDIS/O/234

Juhler, M. • RADSUR/P/176

Just, P. • MOVDIS-T/O/314

1

Kaelin-Lang, A. • MOVDIS/O/140

Kähärä, V. • EPIL/O/277

Kahl, K.・PSYSUR/P/219

Kahlert, U. • BSIDT/F/326

Kalina, M. E EPIL/O/82

Kamadey, O. • PAIN/P/320

Kammermaier, S. • MOVDIS/O/252

Kang, S. W. • RADSUR/P/53

Kang, Y.-N. • RADSUR/P/27

Kaptan, H. • MOVDIS/P/50

Karadag, F. • MOVDIS/P/107

Karamintziou, S. • MOVDIS/F/203

Kasprian, G. • MOVDIS/F/46

Katisko, J. • EPIL/O/277

Kausar, J. • MOVDIS/O/213, MOVDIS/O/214

Kawasaki, T. • MOVDIS/P/199, MOVDIS/P/193

Kayser, S. • PSYSUR/O/293

Keller, C. J. • EPIL/O/266

Khitj, M. • PAIN/O/257

Kho, K. H. • BSIDT/P/60

Kiefer, C. • MOVDIS/O/140

Kim, J.-H. • PAIN/P/265

Kimura, Y. • BSIDT/P/180, MOVDIS/P/193

Kincses, T. Z. • MOVDIS/P/224 


\section{INDEK OF AUTHORS}

Kinfe, T. M. • PAIN/F/96

Király, A. • MOVDIS/P/224

Kiraly, K. • BSIDT/P/307

Klivényi, P. • MOVDIS/P/224

Kmiec, T. · MOVDIS/F/55

Kocabicak, E. • MOVDIS/P/80, MOVDIS/P/81

Kollova, A. • EPIL/P/240

Komoly, S. • MOVDIS/F/122

Kopniczky, Z. • MOVDIS/P/224

Korff, C. • EPIL/O/162

Korfias, S. • BSIDT/O/263, MOVDIS/O/192, MOVDIS/F/299, MOVDIS/F/195, BSIDT/P/264

Kosiara, H. $\cdot$ MOVDIS/F/55

Kostarev, S. • PAIN/P/269

Kouzounias, K. P PAIN/P/204

Kovács, N. • MOVDIS/F/122

Kowalski, M. • BSIDT/O/297

Kozakova, D. • EPIL/P/240

Koziara, H. • MOVDIS/P/54, MOVDIS/P/55

Koziorowski, D. $\cdot$ MOVDIS/P/54

Krack, P. $\cdot$ MOVDIS/O/271

Krainik, A. • PAIN/O/228

Krámská, L. • EPIL/O/82

Kranz, G. · MOVDIS/F/46

Krause-Titz, U. • MOVDIS/O/208, MOVDIS/P/223

Krauss, J. K. • BSIDT/O/102, MOVDIS/O/221, MOVDIS/O/106, MOVDIS/F/101, MOVDIS/F/97, MOVDIS/F/76, MOVDIS/F/77, PSYSUR/O/226, PSYSUR/F/235, MOVDIS/P/222, MOVDIS/P/78, MOVDIS/P/217, PSYSUR/P/219, PSYSUR/P/99

Krolicki, B. • MOVDIS/F/55, MOVDIS/P/54

Krüger, R. $\cdot$ MOVDIS/O/85

Krystkowiak, P. B BSIDT/F/47, MOVDIS/P/48

Ktonas, P. • BSIDT/O/263

Kühn, A. A. P PSYSUR/F/235

Kuhn, J. • PSYSUR/O/110

Kuliffay, Z. • MOVDIS/F/122

Kumada, S. • MOVDIS/P/199

Kunicki, J. • MOVDIS/F/55

Kurt, M. $\cdot$ MOVDIS/P/283

\section{I}

Laghmari, M. • EPIL/O/131, EPIL/O/72

Lambarri, I. • MOVDIS/O/113

Landi, A. • MOVDIS/P/83, MOVDIS/P/61, MOVDIS/P/62

Lanmüller, H. • BSIDT/O/309

Lanteri-Minet, M. PAIN/P/52

Lauterbach, M.•PSYSUR/O/136

Lavano, A. • PSYSUR/O/282

Lavano, S. M. • PSYSUR/O/282

Lavi, A. • MOVDIS/O/51

Lazarewicz, M. • RESIND/O/145, RESIND/O/146, RESIND/O/144

Le Gars, G. • BSIDT/F/47, MOVDIS/P/48

Lee, C.-W. $\cdot$ MOVDIS/P/164 
Lee, J. I. RADSUR/P/53

Lee, K. J. • MOVDIS/P/67, PAIN/P/26, RADSUR/P/27

Lee, M.-K. • MOVDIS/P/185, PAIN/P/183

Lee, S.-B. • PAIN/P/265

Lee, T.-K. • PAIN/P/265

Lee, V. H.-F. • RADSUR/P/171

Lefaucheur, R. • MOVDIS/O/63

Lefranc, M. • BSIDT/F/47, PAIN/O/228, MOVDIS/P/48

Lehtimäki, K. • EPIL/O/277

Lejeune, J.-P. • RADSUR/P/267

Lemaire, J. J. • MOVDIS/P/95, PAIN/P/52, PSYSUR/P/231

Lenartz, D. • PSYSUR/O/110

Lenders, M. • MOVDIS/O/274

Lettieri, C. B BSIDT/F/119

Leveque, M. • PAIN/O/126, MOVDIS/P/57

Levivier, M. • PAIN/O/187, MOVDIS/P/86, RADSUR/P/186

Ley, L. $\cdot$ MOVDIS/F/105

Lezcano, E. • MOVDIS/O/113

Li, D. • PSYSUR/F/281

Lim, L.W. • PSYSUR/O/123

Lima Bezerra Júnior, D. • MOVDIS/F/300, MOVDIS/P/298

Lima, B. • MOVDIS/F/177

Lima, T. P PAIN/F/117

Limousin, P. • BSIDT/F/167, OTHER/P/225

Limousine, P. • PSYSUR/F/205

Lin, G. • PSYSUR/F/281

Lind, G. • PSYSUR/O/202, PAIN/P/204

Lindemann, C. • MOVDIS/F/97

Linder, J. • MOVDIS/O/74

Linderoth, B. • PAIN/O/115, PSYSUR/O/202, PAIN/P/204

Linetzki, E. • MOVDIS/O/51

Linhares, P. • MOVDIS/F/211, MOVDIS/P/303

Liouta, E. • BSIDT/O/263, BSIDT/P/264

Liptai, Z. · MOVDIS/P/190

Liscak, R. • EPIL/O/82, PAIN/O/148

Llanos, S. • MOVDIS/O/87

Lobo-Antunes, J. • BSIDT/O/138

Lönnfors-Weitzel, T. • MOVDIS/O/140

Lopes Alho, A.T. • BSIDT/P/59

Lopes Alho, E. J. • BSIDT/P/58, BSIDT/P/59

Lopes Furlanetti, L. • OTHER/P/287

Lopes, A. • MOVDIS/F/141

López, F. · BSIDT/O/230

López-lbor, J. J. • PSYSUR/P/233

Lopez-Ibor, L. • BSIDT/O/230

López-Ibor, M. I. • PSYSUR/P/233

Lucas Neto, L. • PSYSUR/O/136

Lui, W. M. • RADSUR/P/171

Luis, A. • RADSUR/F/229

Lütjens, G. • MOVDIS/O/221, MOVDIS/F/76, MOVDIS/F/77, PSYSUR/O/226, PSYSUR/F/235, MOVDIS/P/78, MOVDIS/P/222, MOVDIS/P/217, PSYSUR/P/219 
Maarouf, M. • BSIDT/O/302, PSYSUR/O/110, RADSUR/O/301, MOVDIS/P/247

Maciaczyk, D. • BSIDT/F/326

Maciaczyk, J. • BSIDT/F/326

Macron, J. M. • BSIDT/F/47

Mader, I. • BSIDT/P/259

Madureira, L. $\cdot$ RADSUR/F/229

Maedler, B. • PSYSUR/O/293

Magaddino, V. $\cdot$ RADSUR/P/186

Magara, A. • BSIDT/O/297

Maggione, G. • BSIDT/F/253

Magrassi, L. • BSIDT/F/253

Mahmoudzadeh, M. $\cdot$ MOVDIS/P/48

Mahvash, M. • MOVDIS/O/208

Malíková, H. • EPIL/O/82

Maltête, D. • MOVDIS/O/63

Manca, M. $\cdot$ MOVDIS/F/295

Mancini, M. • MOVDIS/F/295

Mandat, T. • MOVDIS/F/55, MOVDIS/P/283, MOVDIS/P/54

Mantione, M. P PSYSUR/O/45

Manu, M. • MOVDIS/O/106

Marc, L. $\cdot M O V D I S / P / 57$

Marceglia, S. • PSYSUR/P/291

Marchetti, M. $\cdot$ RADSUR/O/116

Marchetti, S. • EPIL/F/189

Margalho, P. • MOVDIS/F/141

Margetis, K. • MOVDIS/O/192, MOVDIS/F/299, MOVDIS/F/195

Mariën, P. • BSIDT/F/318

Marmor, O. · MOVDIS/O/51

Marotta, R. • PSYSUR/O/282

Marques, C. • EPIL/F/238

Marras, C. • BSIDT/O/270

Marta, N. • EPIL/O/65, PSYSUR/O/64

Marti-Bonmati, L. · BSIDT/F/153

Martinez-Torres, I. • BSIDT/F/153

Martins, H. • BSIDT/O/236

Massano, J. • MOVDIS/F/211

Massey, L. • MOVDIS/F/56

Matsumoto, S. • BSIDT/P/311

Maurage, C. A. • BSIDT/O/286

Mcgonigal, A. • EPIL/O/72

Medone, M. • RADSUR/O/147

Mehdorn, H. M. • MOVDIS/O/208, MOVDIS/P/223, MOVDIS/P/210

Mehrkens, J. • MOVDIS/O/252

Mehta, A. D. • EPIL/O/266

Mei, L. Z. P PAIN/O/43

Meier, N. · MOVDIS/O/140

Meisner, C. • MOVDIS/O/85

Mendes, A. • MOVDIS/F/177

Menghetti, C. • BSIDT/F/276, PSYSUR/P/285

Merle, P. • BSIDT/F/47 
Messina, G. • BSIDT/O/270, MOVDIS/O/135, PAIN/O/282, PAIN/P/49

Meyerson, B. • PAIN/O/115

Michalik, R. • MOVDIS/F/55

Milanesi, I. • RADSUR/O/116

Mindruta, I. • BSIDT/P/68

Mitchell, R. • MOVDIS/O/213, MOVDIS/O/214

Moens, M. BSIDT/F/318

Moffitt, M. • RESIND/O/206

Mogensen, P. H. • MOVDIS/F/173

Moitinho, M. • MOVDIS/F/141

Molnar, G. • RESIND/O/120, RESIND/O/178, RESIND/O/145, RESIND/O/146

Momjian, S. • EPIL/O/162

Monaco, B. A. • OTHER/P/287

Mondain, M. • MOVDIS/F/198

Mondani, M. • BSIDT/F/119

Monin, H. • BSIDT/P/216

Monteiro, J. • BSIDT/O/236, MOVDIS/F/212. MOVDIS/P/245, PAIN/P/207

Moretti, E. • BSIDT/F/253

Morgado, C. • EPIL/O/142, EPIL/O/246

Mortini, P. • RADSUR/O/147

Morya, E. • MOVDIS/O/220

Moser, D. • BSIDT/O/297

Mossakowski, Z. • MOVDIS/F/32, MOVDIS/P/30, MOVDIS/P/31

Moura De Ribeiro, A.M. • MOVDIS/O/232, MOVDIS/F/198

Moura, A. P PAIN/O/126

Mpoutos, N. • MOVDIS/F/299

Mujic, N. • RESIND/O/111

Müller, R. P. • RADSUR/O/301

Müller-Vahl, K. • PSYSUR/O/226

Mullett, K. • RESIND/O/111

Muracciole, X. • RADSUR/O/128

Mussavi, D. • MOVDIS/O/208

1

Nabais, F. • BSIDT/O/138

Nagy, F. • MOVDIS/F/122

Nagy, P. • PSYSUR/O/244

Nakajima, T. • BSIDT/P/180

Nakano, T. • RADSUR/O/91

Nauman, P. • MOVDIS/F/55, MOVDIS/P/283, MOVDIS/P/54

Navas, M. EPIL/O/65

Negretti, L. • RADSUR/P/186

Nelson, D. • RESIND/O/159

Németh, A. • PSYSUR/O/244

Neto, D. • BSIDT/O/236

Neto, L. • BSIDT/O/236

Neumann, J. • PSYSUR/F/235

$\mathrm{Ng}, \mathrm{S} \cdot \cdot \mathrm{RADSUR} / \mathrm{P} / 171$

Ngoga, D. • MOVDIS/O/213, MOVDIS/O/214

Nguyen, T. B BSIDT/P/259 


\section{INDEK OF AUTHORS}

Nicolelis, M. • MOVDIS/O/220

Nicolini, H. • PSYSUR/F/34

Niedermann, G. • BSIDT/F/326

Nikita, K. • MOVDIS/F/203

Nikkhah, G. B BSIDT/O/161, BSIDT/F/326, BSIDT/P/259, MOVDIS/P/260, RADSUR/P/130, RADSUR/P/261, OTHER/P/287

Noens, B. • BSIDT/O/71, BSIDT/P/69, BSIDT/P/70

Novak, K. · MOVDIS/F/46

Nozaki, A.・RADSUR/O/91

Nuttin, B. • BSIDT/O/278, BSIDT/F/318, PSYSUR/O/279

0

Obszanska, K. • MOVDIS/P/292

Odekerken, V. • MOVDIS/O/274

Oertel, M. • MOVDIS/O/140

Oguro, K. B BSIDT/P/180

Oguzhanoülu, A. • MOVDIS/F/108

Öhman, J. • EPIL/O/277

Okiyama, R. • MOVDIS/P/199, MOVDIS/P/193

Okumura, R. • BSIDT/P/311

Okun, M. S. $\cdot$ MOVDIS/O/121

Oliviero, A. • BSIDT/O/230

Orlov, A. · PAIN/P/269

Ortenzi, A. • EPIL/P/103

Ortiz, T. • PSYSUR/P/233

Østergaard, K. • MOVDIS/F/173, MOVDIS/F/174

Özdemir, M. • PAIN/O/109

Ozieblo, A. • MOVDIS/F/55

Paaschen, S. • MOVDIS/P/223

Paggi, A. • EPIL/P/103

Pall, H. • MOVDIS/O/213, MOVDIS/O/214

Panken, E. • RESIND/O/144

Panourias, I. • MOVDIS/F/299

Papuc, E. • MOVDIS/P/292

Paquis, P. • BSIDT/P/242

Paradzik, V. • BSIDT/F/172

Paris, A. • EPIL/F/189

Park, H. K. • PAIN/P/26

Pastor, J. • EPIL/O/65, PSYSUR/O/64, EPIL/P/155

Peeters, R. R. • BSIDT/P/60

Peltola, J. • EPIL/O/277

Pensantez Orellana, H. • BSIDT/F/253

Pepper, J. • PSYSUR/O/66

Peralta, A. R. • EPIL/O/142, EPIL/O/246

Pereira, R. P PAIN/F/117

Pertovaara, A. • PAIN/O/115

Pfund, Z. · MOVDIS/F/122 


\section{INDEK OF AUTHORS}

Piallat, B. • EPIL/O/237

Pica, A. • MOVDIS/O/140

Piccini, P. • MOVDIS-T/O/315

Pichkur, L. • PAIN/P/201

Pichkur, O. B BSIDT/P/218, PAIN/P/201

Picozzi, P. • RADSUR/O/147

Pimentel, J. • EPIL/O/142, EPIL/O/246

Pinsker, M. • BSIDT/P/259, MOVDIS/P/260, RADSUR/P/130, RADSUR/P/261

Pinto, D. B BSIDT/O/236

Pirillo, D. • MOVDIS/P/83, MOVDIS/P/61, MOVDIS/P/62

Prinz, M. • BSIDT/F/326

Pirker, W. • MOVDIS/F/46

Pistarini, C. B BSIDT/F/253

Plewnia, C. $\cdot$ MOVDIS/O/85

Poelaert, J. • BSIDT/F/318

Poissonet, G. B BSIDT/P/242

Pollo, C. EPIL/O/162, EPIL/O/163, MOVDIS/O/140, BSIDT/P/216, MOVDIS/P/86

Pomposo, I. • MOVDIS/O/113

Pongrácz, F. • BSIDT/P/227

Porta, M. • BSIDT/F/276, PSYSUR/P/285, PSYSUR/P/291

Prabhu, S. E EPIL/O/237

Pralong, E. • MOVDIS/P/57, MOVDIS/P/86

Priori, A. • PSYSUR/P/291

Prinz, M. • BSIDT/F/326

Procházka, T. • EPIL/O/82

Proust, F. • MOVDIS/O/63

Provinciali, L. • MOVDIS/O/98, MOVDIS/F/100, EPIL/P/103

Puers, B. B BSIDT/O/278

Q

Quatrale, R. • MOVDIS/F/295

i.

Raabe, A. • BSIDT/F/139

Racsmány, M. • PSYSUR/O/244

Racz, G. P PAIN/P/304

Rainha Campos, A. • BSIDT/O/138, EPIL/O/142, EPIL/O/246, MOVDIS/F/141, PSYSUR/O/136

Ramirez De Noriega, F. • MOVDIS/O/51

Ramirez, C. B BSIDT/O/286

Ramírez, Y. • MOVDIS/F/248, PSYSUR/F/249, PSYSUR/F/34, BSIDT/P/250, PSYSUR/P/251

Raposo, H. PSYSUR/O/136

Rasche, D. • PAIN/O/44

Raya, S. P PSYSUR/P/251

Regidor, I. • MOVDIS/F/105

Régis, J. • EPIL/O/131, EPIL/O/72, MOVDIS/O/166, MOVDIS/O/271, MOVDIS/F/272, PAIN/O/187, PAIN/O/127, PAIN/O/126, RADSUR/O/128, RADSUR/O/129

Rehncrona, S. • MOVDIS-T/O/316, OTHER/P/225

Reis, C. MOVDIS/F/211 


\section{INDEK OF AUTHORS}

Reis, I. • PAIN/F/117

Reis, N. • MOVDIS/F/212

Reithmeier, T. • BSIDT/P/259, RADSUR/P/130, RADSUR/P/261

Relova-Quinteiro, J. L. • MOVDIS/P/209

Represa, A. • MOVDIS/O/165

Requardt, H. • RADSUR/O/75

Resseguier, N. • PAIN/O/187, PAIN/O/127

Reverberi, C. • PAIN/F/268

Reyes, L. P PSYSUR/P/233

Reyns, N. • BSIDT/O/286, RADSUR/P/267

Rha, H. K. • PAIN/P/26

Ribeiro da Cunha, P. • PAIN/F/117

Ricciuti, R. A. • MOVDIS/O/98, MOVDIS/F/100, EPIL/P/103

Richter, J. • BSIDT/O/90

Rinaldo, S. • BSIDT/F/119

Rito, M. P PAIN/F/117

Rivera, P. • BSIDT/O/230

Rizzi, M. • BSIDT/O/270

Robert, P. • PSYSUR/P/231

Robert, T. • EPIL/O/162

Roche, P. H. • RADSUR/O/129

Rodriguez Oroz, M. C. • MOVDIS/O/84

Rodriguez, O. • MOVDIS/O/113

Roed, H. • RADSUR/P/176

Rogic, M. • BSIDT/F/172

Rola, R. • MOVDIS/F/55, MOVDIS/P/54

Romanelli, P. • EPIL/F/189, RADSUR/O/75

Romero, C. • EPIL/F/238

Rosa, M. • PSYSUR/P/291

Rosa, M. M. • MOVDIS/O/254, MOVDIS/F/255

Rosas, M. J. • MOVDIS/F/211, MOVDIS/P/303

Rossetti, A. • EPIL/O/162, EPIL/O/163

Roujeau, T. • MOVDIS/O/232, MOVDIS/O/104, MOVDIS/F/198, PSYSUR/F/118, PSYSUR/P/92

Roulet, E. • EPIL/O/162

Roussel, P. • PAIN/O/187, PAIN/O/127

Rubio, P. • BSIDT/F/153

Rudas, M. • BSIDT/P/296

Rueff, M.C. PSYSUR/O/136

Ruggeri, L. • BSIDT/F/253

Ruiz De Gopegui, E. • MOVDIS/O/113

Rusinek, M. • BSIDT/P/296 Ruzicka, E. • MOVDIS/F/149

Ryapolova, E.・RESIND/O/143

Rychlicki, F. • MOVDIS/O/98, MOVDIS/F/100, EPIL/P/103

4

Saadé, N. • PAIN/O/115

Saceda, J. • PSYSUR/P/233

Sachdev, B. • BSIDT/F/167

Sadeghi, Y. • MOVDIS/P/86

Saiki, H. • BSIDT/P/311 
Saitou, J. • RADSUR/O/91

Sakas, D. • BSIDT/O/263, BSIDT/P/264, MOVDIS/F/299

Sakas, D. E. • MOVDIS/O/192, MOVDIS/F/195

Sakurai, H. • RADSUR/O/91

Saleh, C. · MOVDIS/O/232

Salgado, L. • PSYSUR/O/136

Salova, E. • MOVDIS/O/312, PAIN/O/257, PAIN/P/258

Sameshima, K. • MOVDIS/O/220

San Martin, E. • MOVDIS/P/247

Sandoval, L. • MOVDIS/F/248, PSYSUR/F/249

Sanrey, E. • MOVDIS/O/104, MOVDIS/F/198, PSYSUR/P/92

Santana, D. • MOVDIS/F/248, PSYSUR/F/249, PSYSUR/F/34, BSIDT/P/250, PSYSUR/P/99

Santos, A. • EPIL/F/238

Sanz, A. B BSIDT/O/230

Sarazin, T. • RADSUR/P/267

Sarem-Aslani, A. • RESIND/O/111

Sarihasan, B. • MOVDIS/P/80

Sarubbo, S. • BSIDT/F/294, MOVDIS/F/295

Saryyeva, A. • MOVDIS/P/222, MOVDIS/P/217, PSYSUR/P/219

Sassi, M. • BSIDT/F/276, PSYSUR/P/285

Satgia, M. • MOVDIS/O/232

Satvrinou, L. • MOVDIS/F/299

Sauer, T. • MOVDIS/F/77

Savart, M. $\cdot$ RESIND/O/111

Sawamoto, N. • BSIDT/P/311

Scerrati, M. • MOVDIS/O/98, MOVDIS/F/100, EPIL/P/103

Schaller, K. • EPIL/O/162

Schechtmann, G. • MOVDIS/O/234, PAIN/P/204

Scheper, G. C. • MOVDIS/P/190

Schkommodau, E. • MOVDIS/P/95

Schlaepfer, T. E. • PSYSUR/O/293

Schmidt, C. P PSYSUR/O/226

Schonfeld, L. • BSIDT/O/124

Schrader, C. • MOVDIS/O/221, PSYSUR/O/226, MOVDIS/P/222, MOVDIS/P/217

Schu, S. • PAIN/F/96

Schüpbach, M. • MOVDIS/O/140

Schuurman, R. • MOVDIS/O/274, PSYSUR/O/45

Schwabe, K. • BSIDT/O/102, MOVDIS/O/106, MOVDIS/F/101, MOVDIS/F/97, PSYSUR/P/219, PSYSUR/P/99

Seeck, M. • EPIL/O/162, EPIL/O/163

Seiger, Å. • PSYSUR/O/202

Seigneuret, E. • PAIN/O/228

Seijo, F. • MOVDIS/P/247

Sensi, M. • MOVDIS/F/295

Sequeira, R. • PSYSUR/O/136

Serramito-Garcia, R. • MOVDIS/P/209

Servello, D. • BSIDT/F/276, PSYSUR/P/285, PSYSUR/P/291

Seto, K. $\cdot$ RADSUR/O/91

Seychelles, A. • MOVDIS/O/232, MOVDIS/O/104, MOVDIS/F/198, PSYSUR/F/118, PSYSUR/P/92

Sganzerla, E. P. • MOVDIS/P/83, MOVDIS/P/61, MOVDIS/P/62

Shabalov, V. • MOVDIS/O/312, MOVDIS/F/273, PAIN/O/257, PAIN/P/258

Shah, A. • MOVDIS/P/95

Sherdil, A. • EPIL/O/237 
Shils, J. • PAIN/O/42, PAIN/O/43

Shin, H.-J. • RADSUR/P/27

Shin, M. • MOVDIS/P/193

Shiokawa, Y. • MOVDIS/P/193

Sierens, D. • BSIDT/O/33

Signorino, M. • EPIL/P/103

Silva, C. MOVDIS/F/177

Silva, J. • RADSUR/F/229

Silva, P. A. • MOVDIS/P/303

Simões Do Couto, F. • PSYSUR/O/136

Sinclair, S. • BSIDT/F/168

Singh, A. · MOVDIS/O/252

Skrap, M. • BSIDT/F/119

Sobstyl, M. • MOVDIS/F/32, MOVDIS/P/29, MOVDIS/P/30, MOVDIS/P/31

Sokal, P. • BSIDT/P/296

Soltan, E. • MOVDIS/F/55, MOVDIS/P/283, MOVDIS/P/54

Song, Z. P PAIN/O/115

Sotelo, M. • BSIDT/P/250

Spehl, T. S. • BSIDT/P/259

Spinelli, L. • EPIL/O/163

Staal, M. · MOVDIS/O/274

Staal, M. J. • MOVDIS/O/290

Stanslaski, S. • RESIND/O/143

Starr, P. • RESIND/O/143

Stavrinou, L • MOVDIS/F/299

Stebbins, G. • BSIDT/O/33

Steinke, K.・RESIND/O/206

Stelmasiak, Z. • MOVDIS/P/292

Stewart, K. • PAIN/P/41

Stieglitz, L. • BSIDT/F/139, MOVDIS/O/140

Stoffregen, W. • RESIND/O/206

Storchi, R. • BSIDT/F/253

Strange, B. • BSIDT/O/230

Stranjalis, G. • BSIDT/O/263

Strojnik, T. • BSIDT/P/319

Sturm, V. • BSIDT/O/302, PSYSUR/O/110, RADSUR/O/301

Stypulkowski, P. $\cdot$ RESIND/O/143

Subrt, O. • MOVDIS/F/149

Sufianov, A. • PAIN/P/269

Sun, B. • PSYSUR/F/281

Sunaert, S. • BSIDT/F/318, BSIDT/P/60

Sunde, N. • MOVDIS/F/173, MOVDIS/F/174

Suzuki, Y. • RADSUR/O/91

Szabó, N. • MOVDIS/P/224

Szalecki, K. • MOVDIS/F/55, MOVDIS/P/54

Szegedi, L. • PAIN/P/304

Székely, G. • MOVDIS/P/194

Szijjártó, G. • MOVDIS/F/122

Szloboda, P. • MOVDIS/P/194 


\section{INDEK OF AUTHORS}

\section{T}

Tachimiri, M. • BSIDT/F/253

Tagaris, G. • MOVDIS/F/203

Tähtinen, T. • EPIL/O/277

Taira, T. • BSIDT/P/180

Talamoni Fonoff, E. • MOVDIS/O/220

Tambuyzer, T. • PSYSUR/O/279

Taniguchi, M. • MOVDIS/P/199, MOVDIS/P/193

Tasnádi, E. • MOVDIS/F/122

Tavares, F. B BSIDT/F/167

Taw, B. B. T. • RADSUR/P/171

Taylor, C. • BSIDT/F/167

Teixeira, J. • PSYSUR/O/136

Teixeira, M. J. • BSIDT/P/58, BSIDT/P/59

Temel, Y. · BSIDT/O/124, MOVDIS/F/125, PSYSUR/O/123, MOVDIS/P/80, MOVDIS/P/81

Temudo, T. • MOVDIS/F/177

Terzi, M. $\cdot$ MOVDIS/P/81

Themistocleous, M. • MOVDIS/O/192, MOVDIS/F/195

Theys, T. · BSIDT/P/60

Thiran, J. P. • BSIDT/P/216

Thomas, D. • MOVDIS/F/56

Thomassin, J. M. • RADSUR/O/129

Thornton, J. • BSIDT/P/306

Tijero, B. $\cdot$ MOVDIS/O/113

Timmermann, L. • MOVDIS/P/247

Toda, H. • BSIDT/P/311

Tomskiy,A. • MOVDIS/O/312, MOVDIS/F/273

Tormasiova, M. • EPIL/P/240

Torres, C.V. • EPIL/O/65, PSYSUR/O/64, EPIL/P/155

Torres, N. • PAIN/O/228

Toscano, T. P PAIN/P/207

Tóth, E. • EPIL/O/266

Touzet, G. • BSIDT/O/286, RADSUR/P/267

Tretyak, I. • BSIDT/P/218

Treuer, H. • BSIDT/O/302, RADSUR/O/301

Trezza, A. • MOVDIS/P/83, MOVDIS/P/61, MOVDIS/P/62

Trippel, M. • RADSUR/P/130, RADSUR/P/261, OTHER/P/287

Tronnier, V. • PAIN/O/44

Trojanowski, T. • MOVDIS/P/292

Tsai, S.-T. • MOVDIS/P/112, MOVDIS/P/164

Tsirogiannis, G. • MOVDIS/F/203

Tsymbaliuk, V. • BSIDT/P/218, PAIN/P/201

Tuleasca, C. P PAIN/O/187, PAIN/O/127, RADSUR/P/186

Tykocki, T. • MOVDIS/F/55, MOVDIS/P/54

1 


\section{INDEK OF AUTHORS}

Ulla, M. • MOVDIS/P/95

Umitsu, Y. • MOVDIS/P/193

Urgosik, D. • MOVDIS/F/149, PAIN/O/148

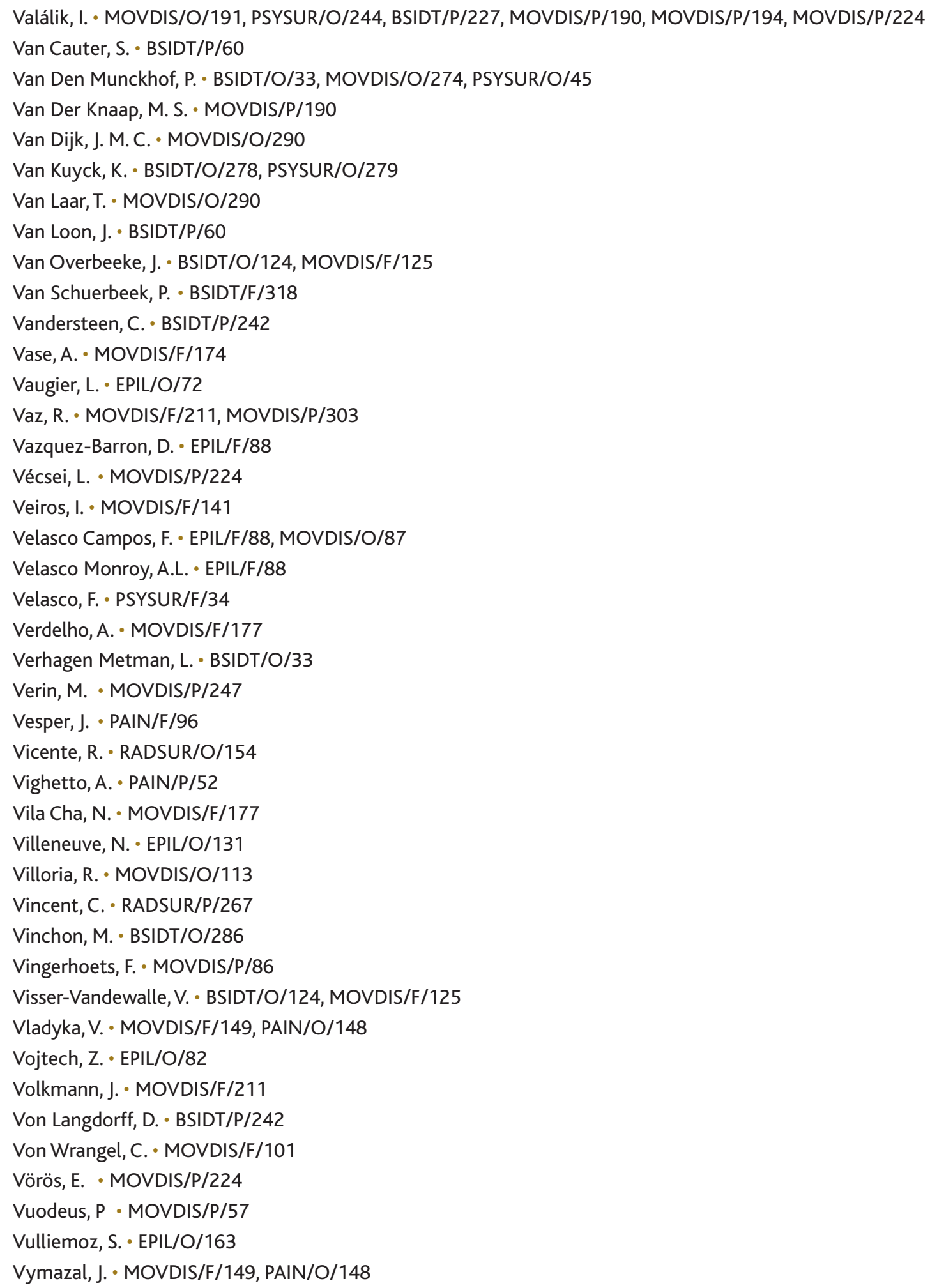




\section{INDEK OF AUTHORS}

Wächter, T. • MOVDIS/O/85

Wahlberg, L. • PSYSUR/O/202

Walach, M. $\cdot$ MOVDIS/O/85

Wallois, F. • MOVDIS/P/48

Wårdell, K. • BSIDT/O/89, BSIDT/O/90, MOVDIS/O/73, PSYSUR/F/205

Warneke, N. • MOVDIS/O/208

Watanabe, E. • BSIDT/P/180

Weber, J. · MOVDIS/O/63

Weiss, D. · MOVDIS/O/85

Wetzalaer, P. • MOVDIS/F/125

White, M. • BSIDT/F/167

Widner, H. • MOVDIS-T/O/317

Wiencke Lund, A. K.・RADSUR/P/176

Wilke, F. PSYSUR/O/226

Wille, C. PAIN/F/96

Winkler, C. B BSIDT/O/161

Winter, L. P PSYSUR/P/219

Witjas, T. • MOVDIS/O/166, MOVDIS/O/271, MOVDIS/F/272

Wloch, A. • MOVDIS/O/221

Wojcik, L. • RESIND/O/206

Wolf, M. • MOVDIS/F/76, MOVDIS/F/77, MOVDIS/P/78

$\mathrm{Wu}, \mathrm{H} . \cdot$ PSYSUR/O/279

Wu, J.・RESIND/O/178, RESIND/O/159

\section{r}

Yaminskiy, Y. • PAIN/P/201

Yáñez, R. • PSYSUR/P/233

Yokochi, F. • MOVDIS/P/199, MOVDIS/P/193

Yokota, H. • BSIDT/P/180

Yomo, S. • RADSUR/O/129

Yoo, D.-S. PAIN/P/265

Younesi, M. O. • BSIDT/F/168

Yousry, T. · MOVDIS/F/56

\section{Z}

Zabek, M. • MOVDIS/F/32, MOVDIS/P/29, MOVDIS/P/30, MOVDIS/P/31

Zaidman, N. • BSIDT/O/286

Zang, Y. P PSYSUR/P/219

Zekaj, E. • BSIDT/F/276, PSYSUR/P/285

Zhan, S. • PSYSUR/F/281

Zhang, X.-X. • PSYSUR/P/281

Zhao, Y. • MOVDIS/P/247

Zhu, C. $\cdot$ RESIND/O/206

Zippo, A. • BSIDT/F/253

Zoia, C. P PAIN/F/268

Zrinzo, L. • BSIDT/F/167, MOVDIS/F/300, MOVDIS/F/56, PSYSUR/F/205, BSIDT/P/306, MOVDIS/P/298

Zsigmond, P. • BSIDT/O/90 